PHYSICAL REVIEW D 93, 089903(E) (2016)

\title{
Erratum: Post-Newtonian cosmological modelling [Phys. Rev. D 91, 103532 (2015)]
}

Viraj A. A. Sanghai and Timothy Clifton

(Received 17 March 2016; published 7 April 2016)

DOI: $10.1103 /$ PhysRevD.93.089903

There were a number of errors in the published version of our paper. These can be corrected by replacing Eq. (71) with

$$
\frac{1}{2} \int_{S} \mathbf{n} \cdot \nabla h_{t t} d A=\int_{\Omega}\left(\frac{1}{2} \nabla^{2} h_{t t}-2 \Phi \nabla^{2} \Phi+|\nabla \Phi|^{2}\right) d V+O\left(\epsilon^{6}\right)
$$

by replacing Eq. (82) with $n^{x(2)}=-\Phi$, and by replacing Eq. (83) with

$$
\kappa \int_{S}\left(\Phi_{, x}+\frac{h_{t t, x}^{(4)}}{2}\right) d S=-4 \pi G M+\frac{1}{2} \int_{\Omega} \nabla^{2} h_{t t}^{(4)} d V^{(0)}+2 \kappa \int_{S} \Phi \Phi_{, x} d S^{(0)}+\kappa \int_{S} X_{, A}^{(2)} \Phi_{, A} d S^{(0)} .
$$

These corrections propagate throughout Sec. V B and the beginning of section VI. First, Eq. (85) should read as

$$
\kappa \int_{S}\left[\Phi_{, x}+\frac{h_{t t, x}^{(4)}}{2}-X_{, A}^{(2)} \Phi_{, A}\right] d S=-4 \pi G M-8 \pi G\left\langle\rho v^{2}\right\rangle-8 \pi G\langle\rho \Phi\rangle-4 \pi G\langle\rho \Pi\rangle-12 \pi G\langle p\rangle .
$$

Then Eq. (89) should read as

$$
\begin{aligned}
A \zeta_{, t t}= & -4 \pi G M+\frac{\kappa S}{\alpha_{\kappa} X^{2}}\left(\frac{96 \pi^{2} G^{2} M^{2}}{\alpha_{\kappa} X}-12 \pi G M C\right)+\kappa \int_{S}\left(\frac{8 \pi G M \Phi}{\alpha_{\kappa} X^{2}}-h_{t x, t}-3 \Phi_{, t} X_{, t}\right) d S-8 \pi G\left\langle\rho v^{2}\right\rangle \\
& -8 \pi G\langle\rho \Phi\rangle-4 \pi G\langle\rho \Pi\rangle-12 \pi G\langle p\rangle+\kappa\left(\frac{112 \pi^{2} G^{2} M^{2}}{\alpha_{\kappa}^{2} X^{5}}-\frac{12 \pi G M C}{\alpha_{\kappa} X^{4}}\right) \int_{S}\left(y^{2}+z^{2}\right) d S+O\left(\epsilon^{6}\right) .
\end{aligned}
$$

Equation (92) should take the following form:

$$
\begin{aligned}
A \zeta_{, t t}= & -4 \pi G M+\frac{\kappa S}{\alpha_{\kappa}\left(X^{(0)}\right)^{2}}\left(\frac{96 \pi^{2} G^{2} M^{2}}{\alpha_{\kappa} X^{(0)}}-12 \pi G M C\right)+\kappa \int_{S}\left(\frac{8 \pi G M \Phi}{\alpha_{\kappa}\left(X^{(0)}\right)^{2}}-3 \Phi_{, t} X_{, t}^{(0)}\right) d S-3 \int_{\Omega} \Phi_{, t t} d V-8 \pi G\left\langle\rho v^{2}\right\rangle \\
& -8 \pi G\langle\rho \Phi\rangle-4 \pi G\langle\rho \Pi\rangle-12 \pi G\langle p\rangle+\kappa\left(\frac{112 \pi^{2} G^{2} M^{2}}{\alpha_{\kappa}^{2}\left(X^{(0)}\right)^{5}}-\frac{12 \pi G M C}{\alpha_{\kappa}\left(X^{(0)}\right)^{4}}\right) \int_{S}\left(y^{2}+z^{2}\right) d S+O\left(\epsilon^{6}\right) .
\end{aligned}
$$

Then the acceleration equation [Eq. (93)] should be given by

$$
\begin{aligned}
X_{, t t}= & -\frac{4 \pi G M}{A}-\frac{12 \pi G M C}{\alpha_{\kappa}\left(X^{(0)}\right)^{2}}-\frac{4 \pi G}{\alpha_{\kappa}\left(X^{(0)}\right)^{2}}\left[2\left\langle\rho v^{2}\right\rangle+2\langle\rho \Phi\rangle+\langle\rho \Pi\rangle+3\langle p\rangle\right] \\
& +\frac{\kappa}{\alpha_{\kappa}\left(X^{(0)}\right)^{2}} \int_{S}\left(\frac{8 \pi G M \Phi}{\alpha_{\kappa}\left(X^{(0)}\right)^{2}}-3 \Phi_{, t} X_{, t}^{(0)}\right) d S-\frac{3}{\alpha_{\kappa}\left(X^{(0)}\right)^{2}} \int_{\Omega} \Phi_{, t t} d V+\frac{1}{\alpha_{\kappa}\left(X^{(0)}\right)^{3}}\left[\frac{96 \pi^{2} G^{2} M^{2}}{\alpha_{\kappa}}\right] \\
& +\left(\frac{112 \pi^{2} G^{2} M^{2}}{\alpha_{\kappa}^{2}\left(X^{(0)}\right)^{5}}-\frac{12 \pi G M C}{\alpha_{\kappa}\left(X^{(0)}\right)^{4}}\right)\left[\frac{\kappa}{\alpha_{\kappa}\left(X^{(0)}\right)^{2}} \int_{S}\left(y^{2}+z^{2}\right) d S-\left(y^{2}+z^{2}\right)\right]+O\left(\epsilon^{6}\right),
\end{aligned}
$$

where $A$ is the total surface area of the cell and it contains both a zeroth-order and an $O\left(\epsilon^{2}\right)$ part. In the specific case of cubic cells, the acceleration equation [Eq. (94)] should be given by 


$$
\begin{aligned}
X_{, t t}= & -\frac{\pi G}{6 \zeta^{2}}\left[M+5 M C+2\left\langle\rho v^{2}\right\rangle+2\langle\rho \Phi\rangle+\langle\rho \Pi\rangle+3\langle p\rangle\right]+\frac{1}{8\left(X^{(0)}\right)^{2}}\left[\int_{S}\left(\frac{4 \Phi \pi G M}{3\left(X^{(0)}\right)^{2}}-6 \Phi_{, t} X_{, t}^{(0)}\right) d S-\int_{\Omega} \Phi_{, t t} d V\right] \\
& +\frac{1}{\left(X^{(0)}\right)^{3}}\left[\frac{7 \pi^{2} G^{2} M^{2}}{27}\right]-\left(\frac{7 \pi^{2} G^{2} M^{2}}{36\left(X^{(0)}\right)^{5}}-\frac{\pi G M C}{2\left(X^{(0)}\right)^{4}}\right)\left(y^{2}+z^{2}\right)+O\left(\epsilon^{6}\right) .
\end{aligned}
$$

For the specific case of regularly arranged pointlike masses at the center of each cell, there is a correction to Eq. (110), which should read as

$$
\begin{aligned}
X_{, t t}= & -\frac{G M}{6 \zeta^{2}}[\pi+5 \pi C-9 E C-3 F C]+\frac{\pi G^{2} M^{2}}{6\left(X^{(0)}\right)^{3}}\left[2 D+\frac{P}{2}-F-3 E+\frac{14 \pi}{9}\right] \\
& -\left(\frac{7 \pi^{2} G^{2} M^{2}}{36\left(X^{(0)}\right)^{5}}-\frac{\pi G M C}{2\left(X^{(0)}\right)^{4}}\right)\left(y^{2}+z^{2}\right)+O\left(\epsilon^{6}\right) .
\end{aligned}
$$

A correction is also required to Eq. (111), which should have read

$$
X_{, t t}=-\frac{G M}{6 X^{2}}[\pi+5 \pi C-9 E C-3 F C]+\frac{\pi G^{2} M^{2}}{6 X^{3}}\left[2 D+\frac{P}{2}-F-3 E+\frac{14 \pi}{9}\right]+O\left(\epsilon^{6}\right) .
$$

This correction propagates though Sec. VII, in the values of the numerical factors in Eqs. (121), (124), and (140). These equations should take the following form:

$$
\begin{gathered}
\mathcal{L}_{, t}^{2} \simeq \frac{16 N}{\mathcal{L}}-\frac{64.9 G^{2} M^{2}}{\mathcal{L}^{2}}-4 C, \\
\left(\frac{d \mathcal{L}}{d \tau}\right)^{2} \simeq \frac{(16 N-54.0 G M C)}{\mathcal{L}}-\frac{4.41 G^{2} M^{2}}{\mathcal{L}^{2}}-4 C(1-3 C) . \\
\left(\frac{d \hat{\mathcal{L}}}{d \tau}\right)^{2} \simeq \frac{(16 N-54.0 G M C)}{\hat{\mathcal{L}}}-\frac{8.06 G^{2} M^{2}}{\hat{\mathcal{L}}^{2}}-4 C(1-3 C) .
\end{gathered}
$$

Finally, Eq. (132) in the published version should have read

$$
\hat{h}_{\hat{t} \hat{t}}^{(2)}=h_{t t}^{(2)}-\frac{a_{, t t}}{a}\left(x^{2}+y^{2}+z^{2}\right)+O\left(\epsilon^{4}\right) .
$$

These corrections, their consequences, and a few smaller typos that have been corrected are all fully incorporated into the latest version of the paper (arXiv:1503.08747v4). 